\title{
Iot Based Physical Condition Screening System For Animals
}

\author{
S. Kalaivani, R.Anitha, S.Anusooya, Jean Shilpa
}

\begin{abstract}
Animal husbandry and its health monitoring is one of the prime facts for the socio-economic development of a nation. This necessitates monitoring the wellbeing of the animals and thereby increasing the food supply of the country. Advancement in wearable biosensors and wireless communication technologies help in ubiquitous E-health monitoring of animals. The proposed system is build up of recent technologies to screen the physical condition of the animals continuously from anywhere and at anytime. The vital parameters like body temperature, heart rate and position tracking are acquired using respective sensors. The collected data are transmitted wirelessly over internet and are stored in a database using IoT technology. The system also alerts the farmers/care takers at the critical conditions. The records that reflect the physical condition of animals collected in the database helps veterinary doctors to provide effective treatment.
\end{abstract}

Keywords - IoT; Arduino; Animal Health monitoring; Sensors

\section{INTRODUCTION}

Animal health plays a vital role in maintaining the stability in agriculture, food supply and to safeguard the economic growth of the country. In order to prevent the economic loss the fitness of the animals is to be monitored. Researches on animal detection and animal health monitoring have been an important field in numerous applications. Several systems have been evolved for monitoring the health condition and tracking the animals [1], [2]. Automated animal health monitoring is one of the important research areas currently being focused by many researchers [3]. Wireless sensor network is the key that combines many new technologies to develop an effective monitoring system [4]. The sensing unit is the main part in the monitoring systems that acquire the required data and also process it for transmission [5] - [6]. Now-a-days there are many sensors like wireless sensors and processing units as Arduino, Rassberry Pi etc. have been developed based on recent technology [7]. This development makes the overall hardware to be simple and movable that suits telemedicine applications. The advancement in wireless communication and have given way for various application to bloom [8].

The most recent development in communication technology is IoT. It integrates various technology and act as a hub

Revised Manuscript Received on July 22, 2019.

S. Kalaivani, Department of Electronics and Communication Engineering, BSA Crescent Institute of Science \& Technology, Chennai, India.

R.Anitha, Department of Electronics and Communication Engineering, BSA Crescent Institute of Science \& Technology, Chennai, India.

S.Anusooya, Department of Electronics and Communication Engineering, BSA Crescent Institute of Science \& Technology, Chennai, India.

Jean Shilpa, Department of Electronics and Communication Engineering, BSA Crescent Institute of Science \& Technology, Chennai, India.

S. Ramachandran, Department of Electronics and Communication Engineering, BSA Crescent Institute of Science \& Technology, Chennai, India. between the interactions of smart devices [9]. The applications of IoT is not limited and can be categorized into heath care, agriculture, smart city, home automation, Smart surveillance, connected cars, industrial internet and so on.

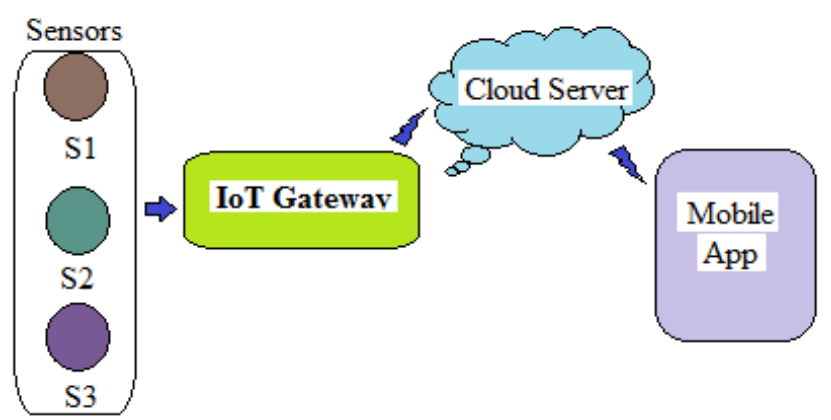

Fig.1. General Architecture of IoT

The general architecture of the IoT system [10] is shown in the above Fig.1. The proposed system is designed based on the IoT system that includes biosensors, Arduino Uno board, GPS, GPRS and GSM for developing an efficient animal health monitoring and animal tracking system. This system will create a database of the vital parameter acquired from the animal in the cloud and also send an alert message to the farmer/care taker on emergency situations.

Proposed System

The block diagram representation of the proposed system is shown in Fig 2 and each module is described below.

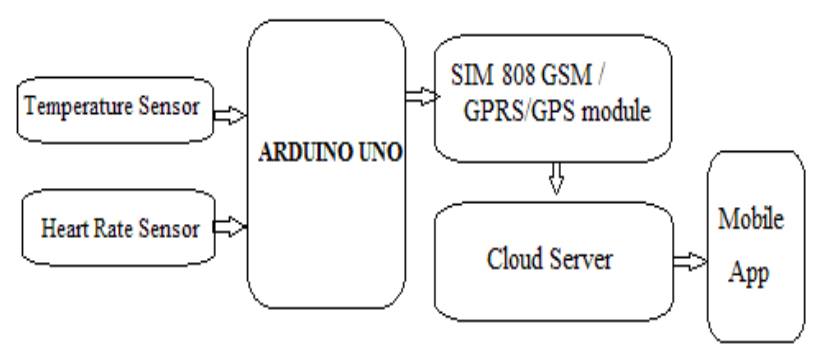

Fig.2 Block diagram of the Proposed System

The various functional units present in the proposed system are: the sensor unit, Arduino Uno board, GSM/GPRS/GPS module, cloud server and mobile App.

(a) Sensor Unit: Temperature and heart rate sensors are interface with the processing board that acquires the body temperature and the heart rate of the animal under test.

(b) Arduino Uno board is used for processing the sensed data in which a processor is built in.

(c) GSM/GPRS/GPS module
Blue Eyes Intelligence Engineering \& Sciences Publication

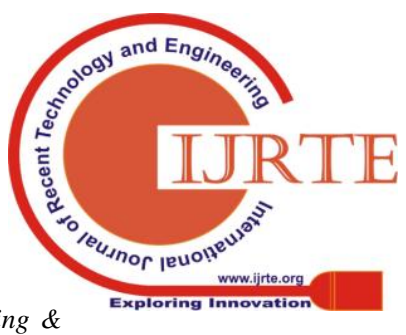




\section{GENYS'ONLINE MARKETING BUYING BEHAVIOUR}

is also interfaced with the Arduino Uno board that helps in tracking the animal and also transmits the data wirelessly to the cloud. The GPRS enables to send SMS to the farmer/zoo officials at any critical situations.

Process Flow Diagram

The step by step process involved in the working of the proposed system is shown diagrammatically using the process flow diagram given below in Fig 3 .

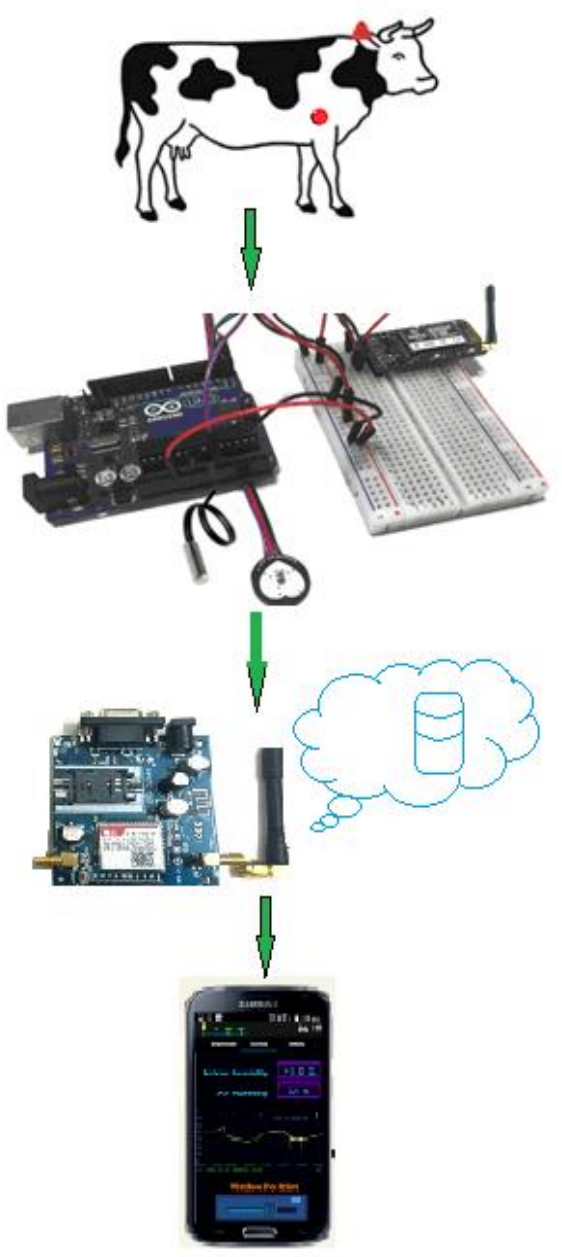

Fig.3 Process Flow diagram

The placement of bio-medical sensors on the animal under test is decided and is placed for ubiquitous screening of their physical condition [11], [12].

Different sensors for monitoring the health condition like body temperature sensor $\&$ heart rate sensor and also a GPS to track the animal are selected interfaced with the processing unit. The processing unit is also interfaced with the wireless module to enable data transmission.

The processed and wirelessly transmitted data is stored in the cloud database over internet by creating an account in thinkspeak.com website.

The data in the cloud database is accessed using the account created through mobile/laptop.

This database will help the zoo keeper/doctors to provide with an effective treatment.

The GSM integrated with the GPRS modem module is used to alert the zoo keeper/doctors on abnormal health conditions of animals.

Hardware design

Temperature Sensor - (DS18b20)

The core body temperature of a domestic animal is always greater than the ambient temperature due to heat generation during metabolism. Every animal has its own normal temperature range with respect to its metabolic activity. The deviation in the body temperature value from its normal range is a sign of the animal not well being. Thermistor is highly sensitive to temperature and could be used to measure the core body temperature of the animals [13]. DS18B20 shown in Fig 4 is the latest temperature sensor that acts like a thermistor. It is a 1-Wire digital temperature sensor, with an output range of $55 \mathrm{oC}$ to $125 \mathrm{oC}(+/-0.5 \mathrm{oC})$ with 9 to 12 -bit precision [14].

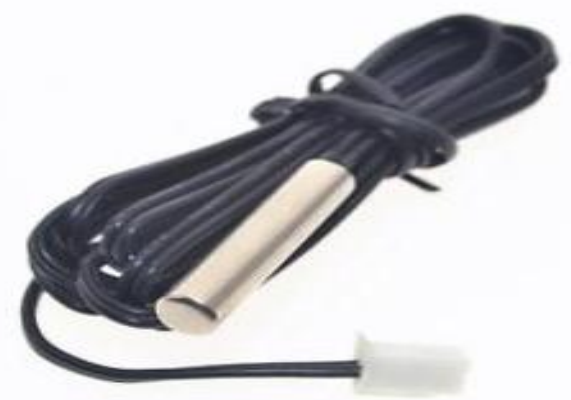

Fig. 4 Temperature sensor - DS18b0

Each sensor has a unique 64-Bit Serial number etched into it and allows a huge number of sensors to be used on one data bus. Thermometer resolution is user-selectable from 9 to 12 bits. The output is a hexadecimal value and so it is divided by 16 to get the temperature value in Celsius. The sensor is accurate and easy to work.

Pulse Sensor

Heart rate (HR) is the number of times heart beats per minute. $\mathrm{HR}$ of an animal is an indicator of its welfare, stress, distress, emotions and excitements. The method for measuring the heart rate is known as photoplethysmography (PPG) [15]. The Pulse Sensor shown in Fig.5 is a photoplethysmography grove ear clip sensor, used for non-invasive heart rate monitoring [16].

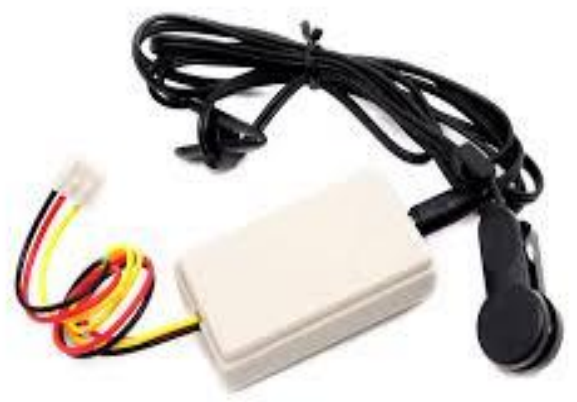

Fig. 5 Pulse sensor

Pulse Sensor Amped responds to relative changes in light intensity. This sensor 
is a plug and play device integrated with the amplification and noise cancellation circuit that makes the measurement simple and fast.

\section{Arduino Uno}

Arduino Uno shown in Fig. 6 is a microcontroller board based on the ATmega328P [17]. It consists of 14 digital I/O pins, 6 analog inputs, a $16 \mathrm{MHz}$ quartz crystal, a USB connection, a power jack, an ICSP header and a reset button.

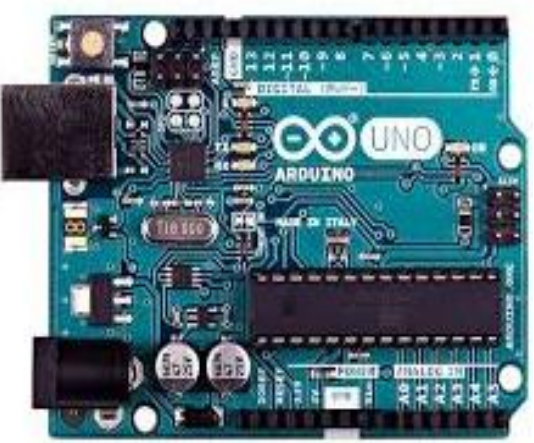

Fig. 6 Arduino Uno board

It can be directly connected to a PC using USB cable and can be powered. It provides easy interfacing and programming platform. Arduino IDE programming platform is free downloadable software. Thus Arduino provides a user friendly environment for the user to implement their applications.

\section{GSM/GPRS/GPS MODULE}

GSM/GPRS module is used for communication between a mobile device and the internet for the IoT application. SIM808 module shown in Fig. 7 is a complete Quad-Band GSM/GPRS module that connects to any GSM network with a 2 G SIM and is also integrated with GPS technology for satellite navigation [18]. The compact design thus helps to send and receive SMS and GPRS data.

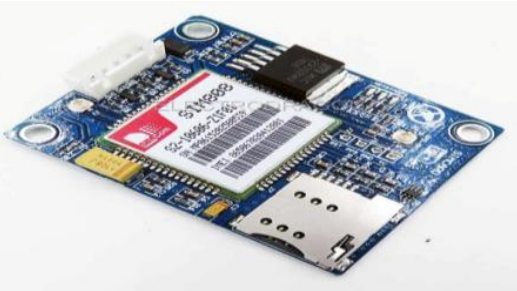

Fig. 7 SIM808 GSM/GPRS/GPS module

Featuring an industry-standard interface and GPS function, allows variable assets to be tracked seamlessly at any location and at anytime with signal coverage.

\section{RESULTS}

The designed physical condition screening hardware is tested on a pet dog and the results are uploaded into a database in an IP by creating an account in Thingspeak.com. Thingspeak.com is an open source IoT platform for maintaining the database [19]. The results obtained using the proposed system on the pet animal is shown in Fig. 8 and Fig.
9. Fig.8 represents temperature data in the chart and Fig.9 shows the database of the same.

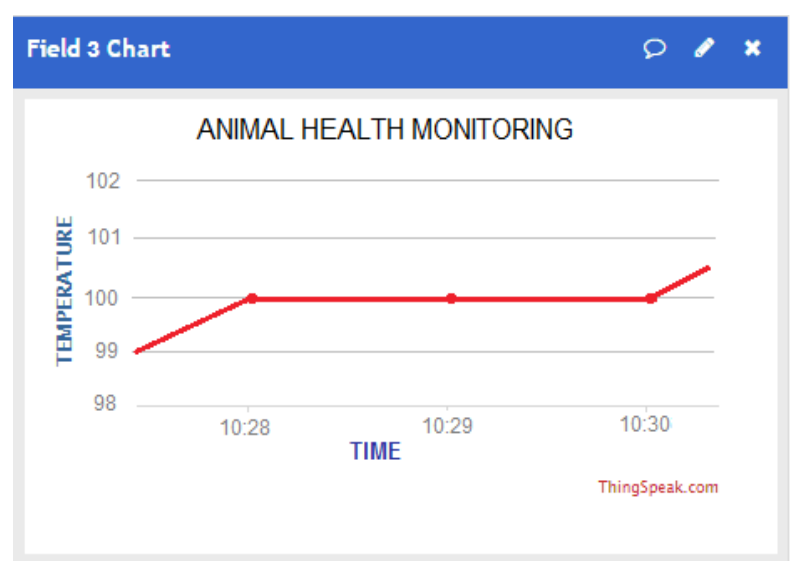

Fig.8 Sensed temperature data chart displayed in thingspeak.com

\begin{tabular}{|r|l|l|l|}
\hline 21 & $2017-10-27 \quad 10: 30: 18$ UTC & 100 & 80 \\
\hline 22 & $2017-10-27$ 10:30:38 UTC & 100 & 80 \\
\hline 23 & $2017-10-27$ 10:30:58 UTC & 100 & 81 \\
\hline 24 & $2017-10-27 \quad 10: 31: 28$ UTC & 100 & 81 \\
\hline 25 & $2017-10-27$ 10:31:48 UTC & 101 & 82 \\
\hline 26 & $2017-10-28$ 11:43:16 UTC & 101 & 82 \\
\hline 27 & $2017-10-2811: 43: 36$ UTC & 101 & 82 \\
\hline 28 & $2017-10-2811: 43: 56$ UTC & 102 & 83 \\
\hline 29 & $2017-10-28$ 11:44:16 UTC & 102 & 83 \\
\hline 30 & $2017-10-2811: 44: 36$ UTC & 102 & 83 \\
\hline
\end{tabular}

Fig. 9 Temperature Database exported from thingspeak.com

This data will be accessible by the concerned authorities of the specified animals.

\section{CONCLUSION}

The prototype designed for screening the vital parameter of the animal under test is simple and user-friendly. Many livestock holders and veterinary doctors will be benefited on using this prototype. They could take care of the animals and monitor them at any time and from anywhere. As a future work wireless sensors could be used to make the system still more comfort for the animals under monitoring.

\section{REFERENCES}

International Journal of Advancements in Research \& Technology, Volum

1. A.Kumar and G.P.Hancke, "Zigbee based Animal health monitoring system" IEEE Sensors Journal, 2013.

2. Greg Byrd, North Carolina State University, "Tracking Cows Wirelessly", IEEE Journals, June 2015

3. Amrutha Helwatkar, Daniel Riordan and Joseph Walsh, "Sensor Technology for animal haelth monitoring", proceedings on 8th international conference on sensing technology, September 2 -4, 2014, Liverpool UK.

4. R. N. Handcock et al., "Monitoring behaviour Environmental interactions 
wireless sensor Networks, GPS collars and satellite remote sensing," Sensors 2009, vol. 9,no. 5, pp. 3586.

5. M. Sveda and R. Vrba, "Integrated smart sensor Networking framework for sensor-based appliances," IEEE Sensors J., vol. 3 , no. 5,pp. 579- 586, Oct. 2003.

6. D.Wobschall, "Networked sensor monitoring using the universal IEEE 1451 Standard", IEEE instrum. Measr. Magazine. 18 -22, April 2008.

7. Leena Narayan, Dr. T. Muthumanickam and Dr. A. Nagappan, "Animal Health Monitoring System using Raspberry Pi and Wireless Sensor",International Journal of Scientific Research and Education (IJSRE), Volume 3 Issue 5, May 2015.

8. Anushka Patil et al., " Smart animal health monitoring", IEEE Explore, 2016.

9. Sajjad Hussain Shah and Ilyas Yaqoob, "A survey: Internet of Things (IOT) technologies, applications and challenges", IEEE Explore, 2016.

10. Pallavi Sethi and Smruti R. Sarangi, "Internet of Things: Architectures, Protocols, and Applications", Journal of Electrical and Computer Engineering, 2017.

11. SureshNeethirajan, "Recent advances in wearable sensors for animal health management", Sensing and Bio-Sensing Research, Elsevier, Volume 12, February 2017, Pages 15-29.

12. Amruta Awasthi et al., "Non-Invasive Sensor Technology for the Development of a Dairy Cattle Health Monitoring System", Computers, MDPI, 2016

13. http://www.biotrack.co.uk/pdf/sensetemp.pdf

14. https://www.sparkfun.com/products/245

15. Rita Brugarolas et al., "Wearable Heart Rate Sensor Systems for Wireless Canine Health Monitoring", IEEE Journal, 2015.

16. http://acid.uclan.ac.uk/doguino

17. https://www.arduino.cc/en/Main/arduinoBoardUno

18. sensorembedded.com/product_extra_files/SIM808.pdf

19. https://thingspeak.com 